\title{
Asociación de polimorfismos del gen Leptina con calidad seminal en toros raza Carora
}

\author{
Association of polymorphisms of the Leptin gene with seminal quality in Carora breed bulls
}

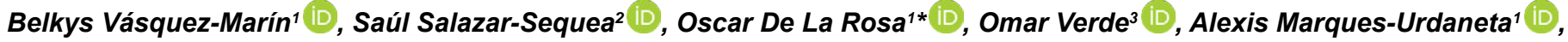 \\ Lourdes Tibisay Vilanova-Fernández ${ }^{4}$
}

\begin{abstract}
${ }^{1}$ Instituto Nacional de Investigaciones Agrícolas, Laboratorio de Biotecnología Agrícola, Maracay, Aragua, Venezuela. ${ }^{2}$ Instituto Nacional de Investigaciones Agrícolas, Laboratorio de Reproducción Animal. Maracay, Aragua, Venezuela. ${ }^{3}$ Universidad Central de Venezuela, Facultad de Ciencias Veterinarias, Cátedra de Genética. Maracay, Aragua, Venezuela. ${ }^{4}$ Universidad Centroccidental Lisandro Alvarado, Facultad de Ciencias Veterinarias, Cátedra de Reproducción Animal. Barquisimeto, Lara, Venezuela.
\end{abstract}

Correo electrónico: delarosa100@gmail.com.

\section{RESUMEN}

La presencia de toros con características seminales deficientes ocasiona fallas considerables en la eficiencia reproductiva de las unidades de producción, debido a la disminución del número de vacas preñadas, siendo más marcado en sistemas bajo programas de inseminación artificial (IA). Los rasgos de calidad seminal (CS) pueden constituir un criterio importante para la selección de machos reproductores utilizados en IA. La identificación de marcadores moleculares asociados con CS en el toro, podría facilitar la selección para estos rasgos. Este estudio tuvo como objetivo evaluar la asociación de los polimorfismos del gen Leptina, sobre la CS de toros de la raza Carora. Se evaluaron las variables volumen de eyaculado (VE), motilidad masal (MM), motilidad individual (MI) y concentración espermática (CE) de 43 toros reproductores Carora, organizados en 8 grupos de edad. Los polimorfismos nucleotídicos simples (SNP) del gen Leptina evaluados fueron: rs29004487 (SNP1), rs29004488 (SNP2), rs29004501 (SNP3) y rs29004508 (SNP4). Se utilizó un análisis de varianza mediante un modelo lineal generalizado (GLM). El factor genotipo contó con 10 niveles y el factor edad, 8 niveles. No se observó efecto del SNP1 sobre ninguna de las variables evaluadas, mientras que el SNP3 tuvo un efecto significativo sobre la CE. Los SNP2 y SNP4 presentaron un efecto altamente significativo sobre la MI y CE. Finalmente, las variables VE y MM no fueron afectadas por ninguno de los SNP estudiados. Los resultados del presente estudio sugieren marcadores potenciales para la valoración y selección genética de bovinos reproductores.

Palabras clave: Alelos; marcadores moleculares; ADN; variación genética; semen

\begin{abstract}
The presence of bulls with poor seminal characteristics causes considerable failures in the reproductive performance of farms, due to decrease in the number of pregnant cows, being more marked in systems under artificial insemination (AI) programs. Seminal quality (SQ) traits can be an important criterion for the selection of breeding males used in Al. The identification of molecular markers associated with SQ in the bull could facilitate the selection for these traits. The objective of this study was to evaluate the association of the polymorphisms of the Leptin gene on the SQ of Carora breeding bulls. The variables ejaculate volume (EV), mass motility (MM), individual motility (IM) and sperm concentration (SC) were evaluated in 43 Carora breeding bulls from an AI Center, organized into 8 age groups. The single-nucleotide polymorphisms (SNPS) of the Leptin gene evaluated were rs29004487 (SNP1), rs29004488 (SNP2), rs29004501 (SNP3) and rs29004508 (SNP4). An analysis of variance was used using a generalized linear model (GLM). The genotype factor had 10 levels and the age factor 8 levels. There was no effect of SNP1 on any of the variables evaluated, while SNP3 had a significant effect on SC. SNP2 and SNP4 showed a highly significant effect on IM and SC. Finally, the variables EV and MM were not affected by any of the SNPs studied. The results of the present study suggest potential markers for the genetic evaluation and selection of breeding cattle.
\end{abstract}

Key words: Alleles; molecular markers; DNA; genetic variation; semen 


\section{INTRODUCCIÓN}

Una eficiencia reproductiva adecuada de los rebaños bovinos (Bos taurus - Bos indicus) constituye un punto clave en el escenario mundial actual y futuro, donde la población humana continuará creciendo [57], anticipando un incremento en la demanda de alimentos del $70 \%$ para el año 2050 [20]. Los esfuerzos para abordar el incremento de la producción de alimentos deberán concentrarse en un uso más eficiente de los recursos [57], con la intensificación sostenible de las ganaderías, que permita la obtención de animales para la producción de carne y leche dirigidos a la alimentación humana.

Entre las estrategias para el incremento sostenible de la producción animal están, el mejoramiento de la fertilidad para aumentar la tasa de nacimientos, así como la introducción y uso de herramientas genéticas basadas en ácido desoxirribonucleico (ADN) y ácido ribonucleico (ARN) para ampliar la capacidad de los programas genéticos en la selección de rasgos individuales y múltiples de modo simultáneo [15]. El rendimiento reproductivo de los rebaños, además de tener implicación en el aporte de alimentos, afecta de manera significativa la rentabilidad de las unidades productivas [18]. Son varios los factores que afectan la eficiencia reproductiva del rebaño y, en este contexto, la fertilidad de los animales y particularmente la del toro es un elemento clave e importante para la sostenibilidad de las ganaderías.

En los sistemas ganaderos que utilizan la monta natural, un toro es utilizado para servir a un número variable de hembras. Este número se incrementa en programas de inseminación artificial (IA) [3]. De lo anterior se deduce que la presencia de machos con características seminales deficientes causará fallas considerables en la eficiencia reproductiva de las unidades de producción [62], debido a la disminución del número de vacas preñadas. Lo mismo fue corroborado en un estudio previo que reportó una correlación significativa entre la tasa de preñez de las vacas y la motilidad masal del semen de los toros, utilizados en programas de IA [52].

El desempeño reproductivo de los toros está influenciado por varios factores tales como: el desarrollo testicular, libido, habilidad para la monta, fortaleza física y calidad seminal (CS) [71]. Este potencial es examinado mediante la evaluación de la solidez reproductiva (BSE, siglas en inglés), que incluye un análisis de rutina del semen para determinar la fertilidad de los toros $[41,59]$. No obstante, el mismo no es capaz de determinar a priori el potencial total de los animales. De igual forma, este análisis convencional de semen no permite la identificación y predicción de los toros subfértiles [19]. Se ha mencionado que hasta el $5 \%$ de los toros en servicio natural pueden ser completamente infértiles y un $30 \%$ adicional puede ser subfértil [71].

Aún con las desventajas de un análisis rutinario de semen para predecir el potencial de un toro reproductor, los rasgos de CS se constituyen en un criterio importante para la selección de machos reproductores utilizados en IA; esto último es crucial para los centros de IA. No obstante, la producción de semen congelado de calidad en condiciones tropicales resulta difícil ya que el $60 \%$ de los toros mestizos (Bos taurus/Bos indicus) son eliminados de los centros de IA, debido a su baja CS, lo que no permite la congelación de su semen [42]. En el caso de los recursos zoogenéticos locales como el Carora, la búsqueda de alternativas para incrementar su productividad de manera sustentable es una elección prioritaria debido a su gran adaptación para producir en ambientes tropicales.
Esta raza es nativa de Carora, estado Lara, Venezuela y proviene del cruce del Criollo Amarillo de Quebrada Arriba y Pardo Suizo. Entre las ventajas de este recurso zoogenético local se cuentan la rusticidad, mansedumbre, fortaleza, vigor, buena reproducción, capacidad de soportar el clima y aprovechar los forrajes tropicales [7].

Se ha observado cada vez con mayor frecuencia, la incorporación de toros jóvenes en los programas de congelación de semen, como consecuencia de su selección temprana lograda mediante herramientas genómicas [49]. De acuerdo a estudios previos, el rendimiento reproductivo de los toros jóvenes presenta una gran variación debido a las diferencias en la edad de inicio de la pubertad dentro y entre razas. Lo anterior representa un desafío para la recolección de semen de alta calidad a partir de estos toros jóvenes [9].

Además de la edad del toro, la CS puede ser afectada por el intervalo de recolección, la frecuencia de recolección, época del año y un amplio rango de factores genéticos y ambientales [56, 64], conjuntamente a una variedad de interacciones entre estos factores [22]. En relación a los factores genéticos, reportes previos demostraron que los rasgos de CS tales como volumen y CE, fueron moderadamente heredables $(0,15$ a 0,30$)$, mientras que la motilidad y el porcentaje de espermatozoides anormales fueron altamente heredables (cercano a 0,60) [26]. Lo anterior indica la oportunidad de mejorar estos rasgos mediante selección asistida por marcadores (SAM) localizados en genes candidatos.

La implicación de los genes sobre el control de rasgos relacionados con CS ha sido estudiada previamente [23, 24, 39]. Los genes candidatos pueden ser utilizados como marcadores alternativos para la selección de toros con parámetros aceptables de CS $[13,14]$. De esta forma, la disponibilidad de información genómica para la detección temprana de machos no aptos para la producción de semen, podría reducir sustancialmente los costos de producción de estos futuros reproductores [65].

El efecto de marcadores moleculares sobre la CS ha sido descrito para carneros (Ovis aries) [8], machos cabríos (Capra hircus) [51], cerdos (Sus scrofa) [43] y toros [40, 46]. De igual forma, se han reportado genes involucrados en la regulación de la motilidad espermática [25], así como asociados con volumen seminal y número total de espermatozoides [26].

Entre los genes estudiados por su posible efecto sobre los rasgos reproductivos en hembras y machos bovinos se encuentra Leptina (LEP). Este gen codifica la hormona del mismo nombre, la cual ha sido implicada en la regulación de la ingesta alimenticia en ovejas y toros $[21,48]$ y como indicador del balance energético durante el periodo peri parto en vacas lecheras [53]. Este gen está localizado en el cromosoma 4 bovino [55] y consiste de dos intrones y tres exones, siendo los exones 2 y 3 los responsables de la traducción de la proteína [28].

Polimorfismos del gen LEP han sido asociados con la concentración de Leptina circulante [30] y con rasgos de importancia económica en diferentes especies, tales como, de producción de leche [54], características de la canal en ganado Nellore [63], calidad de la carne en bovinos y cerdos $[10,66]$, rasgos de crecimiento en cabras y novillos [44, 69], eficiencia alimenticia en toros Nellore [48] y rasgos de fertilidad [29]. En el caso del rendimiento reproductivo del macho, se ha comprobado el efecto de esta hormona sobre la pubertad, espermatogénesis, maduración, y motilidad espermática 
en diferentes especies $[33,45]$ y sobre la capacitación espermática en cerdos y búfalos (Bubalus bubalis) [6, 31]. Se ha demostrado que la Leptina actúa en diferentes niveles del eje hipotálamo-pituitariatestículo, y sobre la célula espermática en sí misma [50], lo que sugiere que esta hormona puede regular de modo directo o indirecto la función espermática.

No obstante, el efecto del gen LEP sobre la función reproductiva del macho bovino y en especial sobre la calidad espermática no ha sido completamente dilucidado, por lo que este estudio tuvo el objetivo de evaluar la asociación de los polimorfismos del gen LEP, sobre la CS de toros de la raza Carora.

\section{MATERIALES Y MÉTODOS}

\section{Datos fenotípicos}

Para la caracterización de la CS se evaluaron las variables VE, MM, MI y CE. Se utilizaron los datos fenotípicos de 43 toros reproductores pertenecientes al Centro de Inseminación Artificial Carora (CIAC), ubicado a 12 kilómetros de la ciudad de Carora, en la carretera Lara-Zulia, municipio Pedro León Torres, estado Lara, Venezuela $\left(10^{\circ} 04^{\prime} 12^{\prime \prime} \mathrm{N} \mid 70^{\circ} 12^{\prime} 27^{\prime \prime} \mathrm{O}\right)$. Este centro se encuentra situado a una altura de 419 metros sobre el nivel del mar (m.s.n.m.), en un ambiente general árido-cálido, con una precipitación promedio anual de 734,68 milímetros ( $\mathrm{mm}$ ), evaporación media anual de $3.058 \mathrm{~mm}$ y una temperatura media anual de $28,2^{\circ} \mathrm{C}$.

Los datos fueron obtenidos de las planillas de control de congelación de semen, recopiladas durante 11 años (2003 - 2014). Se eliminaron 4.592 observaciones por encontrarse incompletas, resultando en una matriz final de 2.182 observaciones para el análisis genético y estadístico.

Las evaluaciones seminales fueron realizadas en diferentes momentos de la vida reproductiva de los toros (en la mayoría de los animales utilizados en este estudio), es decir, las observaciones de cada animal, corresponden a diferentes momentos de su vida como reproductor.

Los grupos de edad definidos en la presente investigación se describen en la TABLA I.

TABLA I

Grupos de edad de la población de toros de raza Carora del CIAC utilizados en el presente estudio

\begin{tabular}{cc}
\hline Grupo & Edad \\
\hline 1 & Hasta 12 meses inclusive \\
2 & Desde 12 meses hasta 24 meses inclusive \\
3 & Desde 25 meses hasta 36 meses inclusive \\
4 & Desde 37 meses hasta 48 meses inclusive \\
5 & Desde 49 meses hasta 60 meses inclusive \\
6 & Desde 61 meses hasta 72 meses inclusive \\
7 & Desde 73 meses hasta 84 meses inclusive \\
8 & Desde 85 meses en adelante \\
\hline
\end{tabular}

\section{Datos genotípicos}

Los polimorfismos nucleotídicos simples (SNP) utilizados en el análisis de asociación con el rasgo fenotípico CS se describen en la TABLA II.

\section{TABLA II}

Polimorfismos del gen Leptina presentes en el grupo de toros reproductores Carora estudiados

\begin{tabular}{cccc}
\hline $\begin{array}{c}\text { Polimorfismo } \\
\text { (sinonimia) }\end{array}$ & Nomenclatura & Genotipos & Frecuencia \\
\hline rs29004487 & & & \\
(Y7F, A252T, & SNP1 (A/T) & AA & 0,95 \\
LepClaI) & & AT & 0,05 \\
& & & \\
rs29004488 & & CC & 0,14 \\
(R25C, C305T, & SNP2 (C/T) & TC & 0,53 \\
R4C, C73T, & & TT & 0,33 \\
LepKpn2I) & & & \\
rs29004501 & SNP3 (C/T) & CC & 0,67 \\
(LepSau3AI) & & TT & 0,26 \\
& & & 0,07 \\
rs29004508 & & CC & 0,88 \\
(A80V, LepHphI, & SNP4 (C/T) & CT & 0,12 \\
A59V) & & & \\
\hline
\end{tabular}

Para la obtención y genotipificación de los SNP1 y SNP3 se utilizó la metodología PCR-RFLP, mientras que para los SNP2 y SNP4 se utilizó un sistema de amplificación con cuatro cebadores refractario a mutaciones denominado TETRA PRIMER PCR [72]. El proceso de obtención y genotipificación fue descrito en detalle en un trabajo previo [60].

\section{Análisis estadístico}

Se utilizó un análisis de varianza (ANAVAR) mediante un modelo lineal generalizado (GLM) debido a que los datos fenotípicos correspondientes a las evaluaciones seminales se encontraban desbalanceados, ya que los mismos fueron tomados del trabajo rutinario de un centro de IA, sin arreglo o diseño experimental.

Cada nivel del factor "genotipo" en el ANAVAR, correspondió a cada par de alelos identificado previamente [60]. De este modo, los SNP1 y SNP4 contaron con 2 niveles, mientras que los SNP2 y SNP3 presentaron 3 niveles (TABLA II). El factor "edad" presentó 8 niveles correspondientes a la edad al momento de evaluación de la muestra, representado en la TABLA I.

Para valorar el efecto del factor genotipo de los animales sobre las características seminales evaluadas, se utilizó un modelo lineal aditivo, con el efecto del toro anidado dentro del factor genotipo.

$$
Y_{i j k}=\mu+\alpha_{i}+\left(\beta_{j}\right) \alpha_{i}+\gamma_{k}+\varepsilon_{i j k}
$$


Donde $Y_{i j k}$ es el valor fenotípico de la CS del genotipo $i$, toro $j$ anidado dentro del genotipo $i$ y grupo de edad $k ; \mu$ es la media poblacional del valor fenotípico de la CS; $\alpha i$ es el efecto fijo del genotipo; $\left(\beta_{j}\right) \alpha_{i}$ es el efecto fijo poligénico del toro $j$ anidado dentro del factor genotipo $i$; $\gamma k$ es el efecto fijo del grupo de edad $k$ y $\varepsilon_{i j k}$ es el efecto residual aleatorio.

Las comparaciones entre las medias ajustadas de los genotipos se realizaron mediante la prueba de Tukey post hoc con un nivel de significancia de 0,05. Para todos los análisis se utilizó el programa Minitab® Statistical Software, versión 17 [47].

\section{RESULTADOS Y DISCUSIÓN}

Los resultados obtenidos del ANAVAR se muestran a continuación en la TABLA III.

Con base en los resultados del ANAVAR, se observó un efecto altamente significativo de las variables grupo de edad y toro sobre todas las características seminales evaluadas en los reproductores de raza Carora.

De igual forma, se evidenció que los genotipos del SNP1 no afectaron ninguna de las variables seminales evaluadas. No obstante, se observaron efectos significativos del SNP3 sobre la CE, mientras que los genotipos de los SNP2 y SNP4 ejercieron un efecto altamente significativo sobre MI y CE.
Las medias ajustadas de las variables volumen del eyaculado y motilidad masal (MM), correspondientes a los genotipos dentro de cada SNP, se describen en la TABLA IV.

No se observaron diferencias significativas entre las medias ajustadas de las variables VE y MM, entre genotipos dentro de cada SNP. Este resultado coincide con un estudio realizado en búfalos en el cual no se detectó correlación entre rasgos como el VE y MM con polimorfismos del gen Leptina [17]. Sin embargo, se ha reportado un efecto significante de algunos genotipos de este gen sobre el VE en verracos [34]. Las medias ajustadas de las variables MI y CE, para los genotipos dentro de cada SNP, se detallan en la TABLA V.

No se observaron diferencias significativas en los valores de las medias ajustadas de MI, entre los genotipos de los SNP1 y SNP3. Sin embargo, algunos genotipos de los SNP2 y SNP4 muestran un desempeño significativamente superior $(P<0,05)$. En el caso del SNP2, los genotipos homocigotos (CC) y heterocigoto (TC) presentan una MI superior, comparado con el homocigoto (TT). Similar hallazgo se evidencia en el homocigoto (CC) del SNP4, comparado al heterocigoto (CT). Lo anterior no es coincidente con lo reportado en búfalos [17]. Sin embargo, se ha observado que la $\mathrm{MI}$ es afectada positivamente en toros Hereford por algunos genotipos del polimorfismo IDVGA-51, que se encuentra estrechamente asociado al gen Leptina [46].

TABLA III

Efecto del factor genético (genotipos) del gen LEP sobre las características seminales en toro de raza Carora

\begin{tabular}{|c|c|c|c|c|c|c|c|c|c|c|c|c|c|}
\hline \multirow{2}{*}{$\begin{array}{l}\text { Fuente de } \\
\text { Variación }\end{array}$} & \multirow{2}{*}{ GL } & \multicolumn{3}{|c|}{$\begin{array}{l}\text { Volumen } \\
\text { eyaculado }\end{array}$} & \multicolumn{3}{|c|}{$\begin{array}{c}\text { Motilidad } \\
\text { masal }\end{array}$} & \multicolumn{3}{|c|}{$\begin{array}{c}\text { Motilidad } \\
\text { individual }\end{array}$} & \multicolumn{3}{|c|}{$\begin{array}{c}\text { Concentración } \\
\text { espermática }\end{array}$} \\
\hline & & $\mathrm{CM}$ & $\mathbf{F}$ & $\mathbf{P}$ & $\mathrm{CM}$ & $\mathbf{F}$ & $\mathbf{P}$ & CM & $\mathbf{F}$ & $\mathbf{P}$ & $\mathrm{CM}$ & $\mathbf{F}$ & $\mathbf{P}$ \\
\hline SNP1 & 1 & 4,36 & 1,31 & 0,25 & 0,03 & 0,06 & 0,80 & 145,68 & 1,97 & 0,24 & 157775 & 0,92 & 0,34 \\
\hline Toros & 40 & 7,36 & 2,21 & 0,00 * & 2,46 & 6,04 & $0,00 *$ & 1397,58 & 13,15 & $0,00 *$ & 3468367 & 20,21 & 0,00 * \\
\hline SNP2 & 2 & 2,75 & 0,82 & 0,44 & 0,73 & 1,79 & 0,17 & 592,72 & 5,58 & 0,00 * & 6718813 & 39,15 & 0,00 * \\
\hline Toros & 39 & 6,94 & 2,08 & 0,00 * & 2,48 & 6,08 & $0,00 *$ & 1417,89 & 13,34 & $0,00 *$ & 3382141 & 19,71 & 0,00 * \\
\hline SNP3 & 2 & 5,25 & 1,57 & 0,21 & 0,53 & 1,30 & 0,27 & 6,91 & 0,07 & 0,94 & 731044 & 4,26 & 0,01 * \\
\hline Toros & 39 & 8,10 & 2,43 & 0,00 * & 2,04 & 5,01 & 0,00 * & 1367,64 & 12,87 & 0,00 * & 3542034 & 20,64 & 0,00 * \\
\hline SNP4 & 1 & 5,43 & 1,63 & 0,20 & 0,05 & 0,12 & 0,73 & 680,25 & 6,40 & 0,01 * & 1756241 & 10,23 & $0,00 *$ \\
\hline Toros & 40 & 7,76 & 2,33 & 0,00 * & 2,28 & 5,59 & 0,00 * & 1194,97 & 11,25 & 0,00 * & 3333156 & 19,42 & 0,00 * \\
\hline Edad & 7 & 98,7 & 29,6 & $0,00 *$ & 1,06 & 2,59 & $0,00 *$ & 1857,12 & 17,48 & $0,00 *$ & 6792461 & 39,58 & $0,00^{*}$ \\
\hline $\mathrm{EE}$ & 21,3 & 3,34 & & & 0,41 & & & 106,26 & & & 171633 & & \\
\hline $\mathrm{R}^{2}$ & & & $18,73 \%$ & & & $11,28 \%$ & & & $22,44 \%$ & & & $32,70 \%$ & \\
\hline
\end{tabular}

EE: error experimental, GL: grados de libertad, CM: cuadrados medios, F: valores F, P: valor de probabilidad. Los superíndices ( ${ }^{\star}$ ) indican significancia estadística $(P<0,05)$ 
TABLA IV

Medias ajustadas para volumen de eyaculado y motilidad masal según genotipos del gen LEP en toros de raza Carora en estudio

\begin{tabular}{cccc|cccc}
\hline Genotipos & $\mathbf{n}$ & Volumen $(\mathbf{m l})$ & Grupos & Genotipos & $\mathbf{n}$ & Motilidad Masal (0-5) & Grupos \\
\hline SNP 1 & & & & SNP $\mathbf{1}$ & & & \\
AT & 203 & $5,01 \pm 0,20$ & A & AA & 1976 & $3,28 \pm 0,03$ & A \\
AA & 1976 & $4,78 \pm 0,08$ & A & AT & 203 & $3,26 \pm 0,07$ & A \\
SNP 2 & & & & SNP $\mathbf{2}$ & & & \\
TC & 992 & $4,85 \pm 0,10$ & A & CC & 350 & $3,34 \pm 0,06$ & A \\
CC & 350 & $4,80 \pm 0,16$ & A & TC & 992 & $3,28 \pm 0,03$ & A \\
TT & 837 & $4,70 \pm 0,10$ & A & TT & 837 & $3,23 \pm 0,04$ & A \\
SNP 3 & & & & SNP 3 & & & \\
TT & 80 & $5,00 \pm 0,22$ & A & TC & 566 & $3,32 \pm 0,04$ & A \\
TC & 566 & $4,90 \pm 0,12$ & A & CC & 1533 & $3,27 \pm 0,03$ & A \\
CC & 1533 & $4,73 \pm 0,09$ & A & TT & 80 & $3,20 \pm 0,08$ & A \\
SNP 4 & & & & SNP 4 & & & A \\
CT & 388 & $4,95 \pm 0,13$ & A & CC & 1791 & $3,28 \pm 3,03$ & A \\
CC & 1791 & $4,77 \pm 0,09$ & A & CT & 388 & $3,26 \pm 0,05$ &
\end{tabular}

Grupos con letras iguales no presentan diferencias significativas

TABLAV

Medias ajustadas para motilidad individual y concentración espermática según genotipos del gen LEP en toros de raza Carora en estudio

\begin{tabular}{cccc|cccc}
\hline Genotipos & $\mathbf{n}$ & $\begin{array}{c}\text { Motilidad } \\
\text { Individual (\%) }\end{array}$ & Grupos & Genotipos & $\mathbf{n}$ & $\begin{array}{c}\text { Concentración } \\
\text { Espermática (mm.espz.) }\end{array}$ & Grupos \\
\hline SNP 1 & & & & SNP 1 & & & \\
AA & 1976 & $78,58 \pm 0,47$ & A & AT & 203 & $1439,56 \pm 45,0$ & A \\
AT & 203 & $77,25 \pm 1,12$ & A & AA & 1976 & $1395,72 \pm 18,8$ & A \\
SNP 2 & & & & SNP 2 & & & \\
CC & 350 & $80,18 \pm 0,91$ & A & CC & 350 & $1620,99 \pm 36,6$ & A \\
TC & 992 & $78,86 \pm 0,55$ & A & TC & 992 & $1409,55 \pm 21,9$ & B \\
TT & 837 & $77,28 \pm 0,59$ & B & TT & 837 & $1283,7 \pm 23,6$ & C \\
SNP 3 & & & & SNP 3 & & & \\
TT & 80 & $78,91 \pm 1,25$ & A & TC & 566 & $1455,95 \pm 27,33$ & A \\
TC & 566 & $78,55 \pm 0,68$ & A & CC & 1533 & $1380,11 \pm 20,0$ & B \\
CC & 1533 & $78,47 \pm 0,50$ & A & TT & 80 & $1349,83 \pm 50,3$ & A \\
SNP 4 & & & & SNP 4 & & & \\
CC & 1791 & $78,76 \pm 0,49$ & A & CC & 1791 & $1410,02 \pm 19,5$ & A \\
CT & 388 & $76,74 \pm 0,76$ & B & CT & 388 & $1307,43 \pm 30,4$ & B \\
\hline
\end{tabular}

Grupos con letras diferentes presentan diferencias significativas $(P<0,05)$ 
La presencia de los genotipos CC y TC (SNP2), así como CC (SNP4), podría favorecer cambios en la movilidad de los espermatozoides. La capacidad del espermatozoide para alcanzar el sitio adecuado y fecundar al ovocito es un factor clave para la fertilidad del macho [68]. Así mismo, se ha demostrado que la MM se correlaciona positivamente con la tasa de preñez en vacas sometidas a programas de IA [52]. De igual forma, el rasgo motilidad espermática es utilizado para predecir el potencial reproductivo de los toros durante la evaluación rutinaria BSE. Estudios precedentes han revisado algunos determinantes genéticos de la motilidad espermática, reportándose un QTL para este rasgo en el cromosoma bovino 4, donde se encuentra el gen Leptina [68].

Con respecto a la CE, no se observaron efectos de los genotipos del SNP1 sobre esta variable. Sin embargo, los genotipos de los SNP2, SNP3 y SNP4, mostraron diferencias entre ellos. En este sentido, el homocigoto (CC) del SNP2 fue significativamente superior a los genotipos heterocigoto (TC) y homocigoto (TT). En lo referente al SNP3, el heterocigoto (TC) mostró mayor rendimiento. Finalmente, en el SNP4 se evidenció un comportamiento superior del genotipo homocigoto (CC), al compararlo con el heterocigoto (CT). Estos resultados difieren de lo reportado en búfalos [17]. No obstante, en cerdos se ha observado el efecto positivo de algunos genotipos de Leptina sobre la CE [34].

Se ha mencionado que la acción de Leptina puede ser ejercida a través de receptores ubicados en el sistema neuroendocrino central [37] y/o a través de receptores tisulares periféricos [32]. El rol de esta hormona sobre las características seminales del macho de diferentes especies no ha sido dilucidado y los efectos descritos en el presente trabajo requieren de una evaluación cuidadosa.

En el presente estudio se detectaron los efectos de tres SNP sobre algunos rasgos de calidad seminal. En el caso del SNP2, algunos genotipos de este marcador disminuyeron la MI y CE. Este polimorfismo se encuentra ubicado en el exón 2 y se considera una variante sin sentido que promueve una transición (C/T). Esta a su vez, ocasiona el cambio de una arginina (CGC) por una cisteína (TGC) en el codón 25 de la proteína [28].

Se ha mencionado que este es un cambio no conservativo que ejerce un efecto funcional sobre la molécula al alterar la estructura de la proteína, bloqueando su capacidad de unirse al receptor [11]. Esto puede provocar cambios importantes en la acción hormonal tanto a nivel del hipotálamo, como directamente sobre las células testiculares [61]. En el primer caso, afectando la secreción de la hormona liberadora de gonadotropinas ( $\mathrm{GnRH})$ por parte de las neuronas del hipotálamo y en consecuencia de las hormonas folículo estimulante (FSH) y luteinizante (LH) desde la pituitaria anterior, afectando finalmente las células de Sertoli y de Leydig. En el segundo caso, ejerciendo efectos sobre la síntesis de testosterona y de la espermatogénesis. No obstante, las posibles consecuencias de este SNP sobre la función biológica de la proteína, fueron evaluadas in silico mediante diferentes herramientas bioinformáticas. En ese estudio se concluyó que los efectos de sus variaciones pueden ser la disminución de la acción de la proteína o incluso, ser tolerada por el organismo [2].

Este SNP ha sido relacionado con diferentes rasgos de importancia económica, tales como el contenido graso de la canal [11], mayor producción de leche e incremento del espesor de la grasa dorsal, así como mayor concentración de Leptina en suero
[1]. Este estudio constituye el primer reporte de su asociación con rasgos de calidad seminal en el toro.

En cuanto al SNP3, en esta investigación se evidenció la disminución de la CE por efecto de uno de sus genotipos. Este marcador se encuentra ubicado en el intrón 2 y promueve una transición C/T [2]. Se ha mencionado que las variaciones en las regiones intrónicas no promueven cambios en la secuencia de aminoácidos de la proteína [2]; no obstante, se ha evidenciado que en las regiones intrónicas pueden encontrarse los sitios de corte y empalme, necesarios para producir los transcritos de ARN mensajero maduros. De igual forma, en los intrones se pueden encontrar secuencias consenso denominadas potenciadores o inhibidores de corte y empalme intrónicos, cuya función es necesaria para incrementar o disminuir la fidelidad en las reacciones de corte y empalme [12]. Se ha reportado que la presencia de variantes en estas regiones intrónicas puede estar implicada en la aparición de proteínas no funcionales debido a la inclusión o exclusión de exones, retención de intrones o la introducción de nuevos sitios de corte y empalme [58]. Este SNP ha sido asociado con elevada producción de leche, rendimiento de la proteína y grasa de la leche y edad al primer parto [67]. Este estudio constituye el primer reporte de su asociación con rasgos de calidad seminal.

En el presente estudio, uno de los genotipos del SNP4 estuvo asociado con la disminución de la MI y CE. Este marcador está ubicado en el exón 3 y promueve una transición T/C [28], la cual resulta en un cambio aminoacídico conservativo en la proteína de valina (GTG) a alanina (GCG). Recientemente se reportó que este SNP tiene efectos deletéreos sobre el sitio de unión III de la molécula de Leptina [2]. Este sitio de unión se encuentra alrededor del N-terminal de Leptina y se extiende desde los residuos 47-140 de la proteína madura. Las variaciones del SNP4 pueden ocasionar un plegamiento inadecuado del N-terminal; además los hallazgos obtenidos de los estudios in silico indican que puede tener consecuencias dañinas sobre la proteína en términos de estructura, función y estabilidad [2]. Lo anterior puede alterar la unión de Leptina a su receptor, tanto a nivel del sistema neuroendocrino central como a nivel de los receptores periféricos.

Estudios realizados con anterioridad en hembras bovinas, reportaron la asociación de este SNP con la disminución del intervalo entre partos, días vacíos, número de inseminaciones por concepción y aumento de la tasa de no retorno [35,36]. Al igual que los SNP2 y SNP3, este estudio constituye el primer reporte de su asociación con rasgos de calidad seminal en el toro.

Al analizar el efecto de los SNP evaluados, es importante resaltar que estos marcadores muestran un alto grado de asociación entre sí [60], particularmente entre los pares SNP1-SNP2, SNP1-SNP3 y SNP1-SNP4. Esto puede indicar que las combinaciones alélicas segregaran de forma conjunta la mayoría de las veces. En este caso, probablemente se esté observando el efecto del SNP4 sobre las características de calidad seminal de los toros Carora y que el resto de las combinaciones alélicas estén segregando conjuntamente con este SNP. Investigaciones realizadas con el semen de diferentes especies evidenciaron la expresión de Leptina en el espermatozoide, lo que sugiere una implicación directa de esta hormona sobre la fisiología espermática. Se ha propuesto que la Leptina puede estar involucrada en la modulación de la disponibilidad del sustrato energético de los espermatozoides durante la capacitación [5]. Otros estudios han destacado la 
Revista Cientifica, FCV-LUZ / Vol. XXXI, N4, 147 - 156, 2021

importancia del metabolismo lipídico para la producción de energía para el espermatozoide [4].

Investigaciones realizadas con semen humano revelaron que Leptina en condiciones in vitro podría aumentar la capacidad fecundante de los espermatozoides a través del incremento de la motilidad y de la reacción del acrosoma [38]. Estudios más recientes [31] demostraron una mayor expresión de Leptina en espermatozoides capacitados in vitro comparados con espermatozoides frescos, lo que reafirma su posible papel en la regulación del proceso de capacitación espermática de búfalos.

Por otra parte, se han evidenciado cambios morfológicos, hormonales y enzimáticos significativos en ratones (Mus musculus) deficientes en Leptina (ob/ob) [45]. Estos animales mostraron alteraciones en el epitelio germinativo, donde se observaron pocas espermatogonias, espermatocitos y espermatidas, así como ausencia de espermatozoides en los túbulos seminíferos. De igual forma se demostró compromiso de la ruta esteroidogénica, con bajos niveles de $3 ß$-hidroxiesteroide deshidrogenasa y de la proteína regulatoria esteroidogénica aguda. En estos ratones también se detectó disminución de la expresión de todos los receptores hormonales involucrados en la función testicular (andrógenos, estrógenos, folículo estimulante, luteinizante, aromatasa, nicotinamida adenina dinucleotido fosfato). De acuerdo a estos investigadores, los cambios en la ruta esteroidogénica enzimática y de las enzimas relacionadas con la actividad espermática pueden promover las fallas en la fertilidad de estos animales.

Aún cuando existe abundante bibliografía que reporta los efectos beneficiosos de Leptina sobre las características seminales en diferentes especies, existen resultados contradictorios. En este sentido, se ha reportado en ratones, efectos adversos sobre los parámetros espermáticos, apoptosis de las células testiculares y posiblemente supresión de la esteroidogénesis testicular, luego de la administración exógena de Leptina [70]. Así mismo, se detectó una correlación inversa entre la expresión de Leptina y la motilidad espermática en toros y búfalos [16].

Las variaciones en el gen Leptina se han asociado con la fertilidad masculina humana [27] y los efectos de los polimorfismos de este gen sobre las características seminales en humanos se han reportado [33]. Estos investigadores demostraron que las frecuencias de los genotipos de Leptina difieren entre hombres fértiles e infértiles. De igual forma, concluyeron el potencial efecto protectivo de algunos genotipos y su posible implicancia en la disminución del riesgo de infertilidad a través del aumento de los contajes espermáticos. No obstante, en búfalos se ha demostrado una asociación negativa entre polimorfismos del gen Leptina y la motilidad espermática post congelación [17].

En el presente estudio se identificaron genotipos del gen Leptina favorables para rasgos de CS en el toro, lo que podría permitir sugerirlos como marcadores potenciales para la valoración y selección genética de bovinos reproductores. No obstante, se requieren estudios adicionales y con diferentes poblaciones vacunas para caracterizar de manera adecuada la solidez de las asociaciones de los polimorfismos en este gen con los rasgos seminales, antes de que este tipo de información genética pueda utilizarse para la toma decisiones de selección animal.

\section{CONCLUSIONES}

Las variables grupo de edad y toro ejercieron un efecto altamente significativo sobre todas las características seminales evaluadas en los reproductores de raza Carora.

Los genotipos del SNP1 no afectaron ninguna de las variables seminales evaluadas.

Los genotipos del SNP3 presentaron un efecto significativo sobre la CE.

Los genotipos de los SNP2 y SNP4 tienen un efecto altamente significativo sobre las variables $\mathrm{MI}$ y $\mathrm{CE}$.

Las variables VE y MM no fueron afectadas por ninguno de los SNP estudiados.

\section{AGRADECIMIENTOS}

Al Centro de Inseminación Artificial Carora (CIAC) y al programa de Estímulo a la Investigación e Innovación (PEII), Proyecto $\mathrm{N}^{\circ}$ 201200674: "Caracterización de las variantes alélicas de genes candidatos implicados en el control de la actividad reproductiva de toros de raza Carora, con fines de selección y mejoramiento genético".

\section{REFERENCIAS BIBLIOGRAFICAS}

[1] ALASHAWKANY, A.; SHAHROUDI, F.; NASSIRY, M.; MOUSSAVI, A.; HEYDARPOUR, M.; SADEGHI, B. Association of SNP in the exon II of leptin gene with milk and reproduction traits in Holstein Iranian cows. Biotechnol. 7 (2): 347-350. 2008.

[2] AL-SHUHAIB, M.B. A comprehensive in silico prediction of the most deleterious missense variants in the bovine LEP gene. Bio Tech. 100(4):429-439. 2019. En línea: https://doi. org/gtqh. 08-07-2021

[3] AMANN, R.P.; DEJARNETTE, J.M. Impact of genomic selection of Al sires on their likely utilization and methods to estimate fertility: a paradigm shift. Theriogenol. 77(5): 795-817. 2012.

[4] AMARAL, A.; CASTILLO, J.; ESTANYOL, J.M.; BALLESCA, J.L.; RAMALHO-SANTOS, J.; OLIVA, R. Human sperm tail proteome suggests new endogenous metabolic pathways. Mol. Cell Proteomics. 12(2): 330-342. 2013.

[5] AQUILA, S.; GENTILE, M.; MIDDEA, E.; CATALANO, S.; MORELLI, C.; PEZZI, V.; ANDO, S. Leptin secretion by human ejaculated spermatozoa. J. Clin. Endocrinol. Metab. 90: 4753-4761. 2005.

[6] AQUILA, S.; RAGO, V.; GUIDO, C.; ZUPO, S.; CASABURI, I.; CARPINO, A. Leptin and leptin receptor in pig spermatozoa: evidence of their involvement in sperm capacitation and survival. Reprod. 136(1): 23-32. 2008.

[7] ASOCRICA. Raza Carora. En línea: https://www.razacarora. com.vel. 20/08/20.

[8] BAKHTIAR, R.; ABDOLMOHAMMADI, A.; HAJARIAN, H.; NOKOUSEFAT, Z.; KALANTAR-NEYESTANAKI, D. Identification of g.170G $>A$ and g.332G $>$ A mutations in exon 3 of leptin gene (Bcnl and Cail) and their association with semen quality and testicular dimensions in Sanjabi rams. Anim. Reprod. Sci. 179: 49-56. 2017. 
[9] BARTH, A.D.; BRITO, L.F.; KASTELIC, J.P. The effect of nutrition on sexual development of bulls. Theriogenol. 70 : 485-494. 2008.

[10] BIŽIENĖ, R.; MORKŪNIENĖ, K.; MIŠEIKIENE், R.; PEČIULAITIENĖ, N.; MAKŠTUTTIENĖ, N.; ŠLYŽIUS, E. Effect of single nucleotide polymorphism markers on the carcass and fattening traits in different pig populations. J. Anim. Feed Sci. 27(3): 255-272. 2018

[11] BUCHANAN, F.; FITZSIMMONS, C.J.; VAN KESSEL, A.G.; THUE, T.D.; WINKELMA-SIM, D.C.; SCHMUTZ, S.M. Association of a missense mutation in the bovine leptin gene with carcass fat content and leptin mRNA levels. Genet. Selec. Evolut. 34:105-16. 2002.

[12] CHEN, M.; MANLEY, J.L. Mechanisms of alternative splicing regulation: insights from molecular and genomics approaches. Nat. Rev. Mol. Cell Biol. 10:741-54. 2009

[13] DEB, R.; KUMAR, S.; SINGH, U.; TYAGI, S.; MANDAL, D.K.; SENGAR, G.; SINGH, R.; KUMAR, M.; SHARMA, A. Evaluation of three bovine $Y$ specific microsatellite loci as an alternative biomarkers for semen quality traits in crossbred bull. Anim. Reprod. Sci. 142(2): 121-125. 2013.

[14] DEB, R.; SINGH, U.; RAJA, T.V.; KUMAR, S.; TYAGI, S.; ALYETHODI, R.R.; ALEX, R.; SENGER, G.; SHARMA, S. Designing of an artificial neural network model to evaluate the association of three combined $Y$ specific microsatellite loci on the actual and predicted post thaw motility in crossbred bull semen. Theriogenol. 83(9): 1445-1450. 2015.

[15] DERNER, J.D.; HUNT, L.; EUCLIDES FILHO, K.; RITTEN, J.; CAPPER, J.; HAN, G. Livestock production Systems. In: Rangeland Systems. Processes, management and challenges. Briske, D. (Ed.) Springer: Cham, Suiza. Pp. 367. 2017.

[16] DHANOA, J.K.; MUKHOPADHYAYY, C.S.; ARORA, J.S.; KAUR, S. Expression profile of Leptin and Aromatase-P450 genes and association with sperm motility in fresh bovine ejaculates. Proc. Natl. Acad. Sci. India. 86: 611-616. 2016.

[17] DILBAR, G.H.; QURESHI, Z.I.; BABAR, M.E.; JAMIL, H.; JAVED, M.T. R159Q polymorphism in Leptin gene and its correlation with semen quality parameters in Nili-Ravi buffalo bulls. Pak. Vet. J. 39(1): 61-65. 2019.

[18] ENGELKEN, T.; TREJO, C.; VOSS, K. Reproductive health programs for beef herds: analysis of records for assessment of reproductive performance. In: Youngquist, R. S; Threlfall, W. R. (Eds.). Current therapy in large animal theriogenology. 2nd Ed. Saunders, Philadelphia. USA. Pp. 490-496. 2007.

[19] FAIR, S.; LONERGAN, P. Review: understanding the causes of variation in reproductive wastage among bulls. Anim. 12(s1): 53-62. 2018.

[20] FOOD AND AGRICULTURE ORGANIZATION OF THE UNITED NATIONS (FAO). How to feed the world: Global Agriculture Towards 2050. 2009: Roma. En línea: https://bit. ly/3BzRiyx. 14/08/20.
[21] FOOTE, A.P.; TAIT JR, R.G.; KEISLER, D.H.; HALES, K.E.; FREETLY, H.C. Leptin concentrations in finishing beef steers and heifers and their association with dry matter intake, average daily gain, feed efficiency and body composition. Domest. Anim. Endocrinol. 55: 136-141. 2016.

[22] FOOTE, R.H. Factors influencing the quantity and quality of semen harvested from bulls, rams, boars and stallions. J. Anim. Sci. 47: 1-11. 2014.

[23] FORTES, M.R.; REVERTER, A.; HAWKEN, R.J.; BOLORMAA, S.; LEHNERT, S.A. Candidate genes associated with testicular development sperm quality and hormone levels of inhibin, luteinizing hormone and insulin-like growth factor 1 in Brahman bulls. Biol. Reprod. 87: 58. 2012. En línea: https://doi.org/gtqg. $12 / 08 / 20$

[24] FORTES, M.R.; REVERTER, A.; KELLY, M.; MCCULLOCH, R.; LEHNERT, S.A. Genome-wide association study for inhibin, luteinizing hormone, insulin-like growth factor 1, testicular size and semen traits in bovine species. Androl. 1: 644-650. 2013.

[25] HERING, D.M.; OLENSKI, K.; KAMINSKI, S. Genome-wide association study for poor sperm motility in Holstein-Friesian bulls. Anim. Reprod. Sci. 146: 89-97. 2014a.

[26] HERING, D.M.; OLENSKI, K.; RU, S.A.; KAMINSKI, S. Genomewide association study for semen volume and total number of sperm in Holstein-Friesian bulls. Anim. Reprod. Sci. 151: 126-130. 2014b.

[27] HODŽIĆ, A.; RISTANOVIĆ, M.; ZORN, B.; TULIĆ, C.; MAVER, A.; NOVAKOVIĆ, I.; PLASESKA-KARANFILSKA, D.; PETERLIN, $B$. Genetic variation in leptin and leptin receptor genes as a risk factor for idiopathic male infertility. Androl. 5(1): 70-74. 2016.

[28] HUNT, S.; MCLAREN, W.; GIL, L.; THORMANN, A.; SCHUILENBURG, H.; SHEPPARD, D.; PARTON, A.; ARMEAN, I.; TREVANION, S.; FLICEK, P.; CUNNINGHAM, F. Ensembl variation resources. Database. 18: 1-12. 2018. En línea: https:// doi.org/gtaj

[29] JECMINKOVA, K.; MÜLLER, U.; KYSELOVA, J.; SZTANKOOVA, Z.; ZAVADILOVA, L.; STIPKOVA, L.; MAJZLIK, I. Association of leptin, toll-like receptor 4 and chemokine receptor of interleukin $8 \mathrm{C}-\mathrm{X}-\mathrm{C}$ motif single nucleotide polymorphisms with fertility traits in czech Fleckvieh cattle. Asian-Australas. J. Anim. Sci. 31(11): 1721-1728. 2018. En línea: https://doi.org/gdbgkp

[30] JONAS, E.; MARTIN, G.B.; CELI, P.; SOATTIN, M.; THOMSON, P.C.; RAADSMA, H.W. Association of polymorphisms in leptin and leptin receptor genes with circulating leptin concentrations, production and efficiency traits in sheep. Small Rumin. Res. 136: 78-86. 2016. En línea: https://doi.org/f8ndw2

[31] KANCHAN, K.; AMAN, K.; VISHAL, S.; ANUPAMA, K.; TRILOK, N. Leptin gene transcripts study in vitro capacitated spermatozoa of buffalo bull semen. Haryana. Vet. 53(1): 50-54. 2014.

[32] KHAKI, A.; BATAVANI, R.A.; NAJAFI, G. The in vitro effect of leptin on semen quality of water buffalo (Bubalus bubalis) bulls. Vet. Res. Forum. 41(1): 7-12. 2013. 
Revista Cientifica, FCV-LUZ / Vol. XXXI, N4, 147 - 156, 2021

[33] KHOSROPOUR, S.; HAMIDI, M.; FATTAHI, A.; KHODADATI, I.; KARAMI, M.; FAZILATI, M. Leptin and leptin-receptor polymorphisms in fertile and infertile men. Syst. Biol. Reprod. Med. 63(1): 7-14. 2016.

[34] KMIEĆ, M.; KULIG, H.; KONIK, A. Preliminary reproduction performance traits of boars results on associations between leptin gene (LEP) and some reproduction performance traits of boars. Arch. Tierz. 46: 63-70. 2003.

[35] KOMISAREK, J.; ANTKOWIAK, I. The relationship between leptin gene polymorphisms and reproductive traits in Jersey cows. Pol. J. Vet. Sci. 10 (4): 193-197. 2007.

[36] KOMISAREK, J. Impact of LEP and LEPR gene polymorphisms on functional traits in Polish Holstein-Friesian cattle. Anim. Sci. Pap. Rep. 28:133-141. 2010.

[37] KUL, A.; BALTACI, A.K.; MOGULKOC, R. Effect of testosterone supplementation on leptin release in rats after castration and/ or unilateral surrenalectomy. Endokrynol. Pol. 63(2): 119-124. 2012.

[38] LAMPIAO, F.; DU PLESSIS, S.S. Insulin and leptin enhance human sperm motility, acrosome reaction and nitric oxide production. Asian J. Androl. 10(5): 799-807. 2008.

[39] LIU, J.; SUN, Y.; YANG, C.; ZHANG, Y.; JIANG, Q.; HUANG, J.; JU, Z.; WANG, X.; ZHONG, J.; WANG, C. Functional SNPS of INCENP affect semen quality by alternative splicing mode and binding affinity with the target BTA-miR-378 in chinese Holstein bulls. PloS One. 11(9): e0162730. 2016.

[40] LIU, X.; JU, Z.; WANG, L.; ZHANG, Y.; HUANG, J.; LI, Q.; LI, J.; ZHONG, J.; AN, L.; WANG, C. Six novel single nucleotide polymorphisms in SPAG11 gene and their association with sperm quality traits in Chinese Holstein bulls. Anim. Reprod. Sci. 129: 14-21. 2011.

[41] LONE, S.A.; PARAY, A.R.; MIR, S.H.; GANAIE, B.A.; SINHA, R.; SINGH, P. Breeding soundness evaluation in bulls: A review. Biomed. J. Sci. Tech. Res. 1(5): 1-4. 2017.

[42] MANDAL, D.K.; KUMAR, M.; TYAGI, S. Effect of age on spermiogram of Holstein Friesian $\times$ Sahiwal crossbred bulls. Anim. 4(4): 595-603. 2009.

[43] MAŃKOWSKA, A.; BRYM, P.; PAUKSZTO, Ł.; JASTRZĘBSKI, J.P.; FRASER, L. Gene polymorphisms in boar spermatozoa and their associations with post-thaw semen quality. Int. J. Mol. Sci. 21(5): 1-30. 2020.

[44] MARTÍNEZ, R.; ROCHA, J.F.; BEJARANO, D.; GÓMEZ, Y.; ABUABARA, Y.; GALLEGO, J. Identification of SNPs in growthrelated genes in colombian creole cattle. Genet. Mol. Res. 15(3): 1-16. 2016.

[45] MARTINS, F.F.; AQUILA, M.B.; MANDARIM-DE-LACERDA, C.A. Impaired steroidogenesis in the testis of leptin-deficient mice (ob/ob-/-). Acta Histochem. 119(5): 508-515. 2017.

[46] MENEGASSI, S.R.; PEREIRA, G.R.; AGUIAR, P.R.; PEREIRA, K.S.; KOETZ JUNIOR, C.; BRACCINI NETO, J.; PERIPOLLI, V.; BERLITZ, C.G.; BARCELLOS, J.O. Candidate genes related to reproductive traits of Hereford and Braford bulls. Sémin. Cien. Agrar. 39(3): 1335-1350. 2018.
[47] MINITAB. Users Guide. 2010. En línea: www.minitab.com. $10 / 01 / 20$.

[48] MOTA, L.F.; BONAFÉ, C.M.; ALEXANDRE, P.A.; SANTANA, M.H.; NOVAIS, F.J.; TORIYAMA, E.; PIRES, A.V.; SILVA, S.; LEME, P.R.; FERRAZ, J.B.; FUKUMASUS, H. Circulating leptin and its muscle gene expression in Nellore cattle with divergent feed efficiency. J. Anim. Sci. Biotechnol. 8(71): 1-5 2017.

[49] MURPHY, E.M.; KELLY, A.K.; O'MEARA, C.; EIVERS, B.; LONERGAN, P.; FAIR, S. Influence of bull age, ejaculate number, and season of collection on semen production and sperm motility parameters in Holstein Friesian bulls in a commercial artificial insemination center. J. Anim. Sci. 96(6): 2408-2418. 2018.

[50] NIKBAKHT, G.; ALI MEHR, M.R.; BAGHBANZADEH, A.; TAJIK, P.; TAMANINI, C.; EMAM, M. Leptin receptor mRNA in bull ejaculated spermatozoa. Reprod. Domestic. Anim. 45: 237-242. 2010.

[51] NIKBIN, S.; PANANDAM, J.M.; YAAKUB, H.; MURUGAIYAH, M.; SAZILI, A.Q. Novel SNPs in heat shock protein 70 gene and their association with sperm quality traits of Boer goats and Boer crosses. Anim. Reprod. Sci. 146(3-4): 176-181. 2014.

[52] NOGUEIRA, É.; RODRIGUES, W.B.; SILVA, C.S.; COSTA E SILVA, V.; POTIENS, J.R.; SILVA, J.C.; SUTOVSKY, P. Identification of seminal parameters predictive of conception rates in Angus and Nelore bulls used in TAI. Proceedings of the International Bull Fertility Conference. Westport. 27-30/05. Irlanda. Pp. 324. 2018.

[53] NOWROOZI-ASL, A.;AARABI, N.; ROWSHAN-GHASRODASHTI, A. Ghrelin and its correlation with leptin, energy related metabolites and thyroidal hormones in dairy cows in transitional period. Pol. J. Vet. Sci. 19(1): 197-204. 2016.

[54] PEGOlO, S.; CECCHINATO, A.; MELE, M.; CONTE, G.; SCHIAVON, S.; BITTANTE, G. Effect of candidate gene polymorphisms on the detailed fatty acids profile determined by gas chromatography in bovine milk. J. Dairy Sci. 99: 45584573. 2016.

[55] PERUCATTI, A.; DIMEO, G.P.; VALLINOTO, M.; KIERSTEIN, G.; SCHNEIDER, M.P.; INCARNATO, D.; CAPUTI, A.; MOHAMMADI, G.; VONGHIA, G.; SILVA, G.; BRENIG, B.; IANUZZI, L. FISH-mapping of LEP and SLC26A2 genes in sheep, goat and cattle R-banded chromosomes: comparison between bovine, ovine and caprine chromosome 4(BTA4/OAR4/ $\mathrm{CHI} 4$ ) and human chromosome 7 (HSA7). Cytogenet. Genome Res. 115: 7-9. 2006.

[56] PERUMAL, P. Scrotal circumference and its relationship with testicular growth, age and body weight in Tho Tho (Bos indicus) bulls. Int. Sch. Res. Notices. 2014: 1-6. 2014.

[57] PORKKA, M.; GERTEN, D.; SCHAPHOFF, S.; SIEBERT, S.; KUMMU, M. Causes and trends of water scarcity in food production. Environ. Res. Lett. 11(1): 1-13. 2016.

[58] RAMÍREZ-BELLO, J.; VARGAS-ALARCÓN, G.; TOVILLAZÁRATE, C.; FRAGOSO, J.M. Polimorfismos de un solo nucleótido (SNP): implicaciones funcionales de los SNP reguladores (rSNP) y de los SNP-ARN estructurales (srSNP) en enfermedades complejas. Gac. Med. Mex. 149: 220-228. 2013. 
[59] RODRÍGUEZ-MARTÍNEZ, H. Semen evaluation technique and their relationship with fertility. Anim. Reprod. 10(3): 148-159. 2013.

[60] SALAZAR, S.; DE LAROSA, O.; MARQUES, A.; VILANOVA, L.; VÁSQUEZ, B. Caracterización de polimorfismos del gen Leptina en sementales de la raza Carora. Rev. Científ. FCV-LUZ. XXX(2): 32-43. 2020.

[61] SENGUPTA, P.; BHATTACHARYA, K.; DUTTA, S. Leptin and male reproduction. Asian Pac. J. Reprod. 8(5):220-226. 2019.

[62] SHOJAEI, H.A.; CLAUDE, R. Modern reproductive technologies and breed improvement. In: The genetics of cattle, Garrick, D; Ruvinsky, A. (Eds.). lowa State University, lowa, Estados Unidos. Pp. 284-317. 2015.

[63] SILVA, D.B.; CRISPIM, B.A.; SILVA, L.E.; OLIVEIRA, J.A.; SIQUEIRA, F.; SENO, L.O.; GRISOLIA, A.B. Genetics variations in the leptin gene associated with growth and carcass traits in Nellore cattle. Genet. Mol. Res. 13(2): 3002-3012. 2014.

[64] SNOJ, T.; KOBAL, S.; MAJDIC, G. Effects of season, age and breed on semen characteristics in different Bos taurus breeds in 31-year retrospective study. Theriogenol. 79:847-852. 2013.

[65] THUNDATHIL, J.C.; DANCE, A.L.; KASTELIC, J.P. Fertility management of bulls to improve beef cattle productivity. Theriogenol. 86(1):397-405. 2016.

[66] TIAN, J.; ZHAO, Z.; ZHANG, L.; ZHANG, Q.; YU, Z.; LI, J.; YANG, R. Association of the leptin gene E2-169T>C and E3-299T>A mutations with carcass and meat quality traits of the chinese Simmental-cross steers. Gene. 518: 443-448. 2013.
[67] TRAKOVICKÁ, A.; MORAVČIKOVÁ, N.; KASARDA, R. Genetic polymorphisms of leptin and leptin receptor genes in relation with production and reproduction traits in cattle. Acta Biochim. Pol. 60 (4): 783-787. 2013

[68] VALOUR, D.; MICHOT, P.; EOZENOU, C.; LEFEBVRE, R.; BONNET, A.; CAPITAN, S.; UZBEKOVA, E.; SELLEM, E.; PONSART, C.; SCHIBLER, L. Dairy cattle reproduction is a tightly regulated genetic process: Highlights on genes, pathways, and biological processes. Anim. Front. 5(1): 32-41. 2015.

[69] WANG, C.; ZHANG, H.; NIU, L.; GUO, J.; JIA, X.; WANG, L.; LI, L.; ZHANG, H.; ZHONG, T. The novel SNPs of leptin gene and their associations with growth traits in chinese Nanjiang yellow coat. Gene. 572(1): 35-41. 2015.

[70] WANG, X.; ZHANG, X.; HU, L.; LI, H. Exogenous leptin affects sperm parameters and impairs blood testis barrier integrity in adult male mice. Reprod. Biol. Endocrinol. 55(16): 1-11. 2018.

[71] WATHES, D.C.; DISKIN, M.G., Reproduction, events and management: Mating management: Fertility. Reference Module in Food Science. 1-11 2016. En línea: https://doi. org/gnnk. 11/07/20.

[72] YE, S.; DHILLON, S.; KE, X.; COLLINS, A.R.; DAY, I.N. An efficient procedure for genotyping single nucleotide polymorphisms. Nucleic Acids Res. 29(17): e88. 2001 